\title{
Carcinoma of the breast in men
}

\author{
Shannon Barry, MD, Kelli Y. Ha, MD, and Louba Laurie, MD
}

Male breast cancer is an uncommon disease of uncertain etiology. We describe a 66-year-old man who presented with a palpable mass in the left breast with associated nipple inversion. Mammographic images demonstrated a spiculated mass within the subareolar left breast at the palpable area of concern. Sonographic evaluation demonstrated a hypoechoic mass within the subareolar left breast at the location of the mammographic abnormality. The patient underwent an excisional biopsy and was subsequently diagnosed with high-grade invasive ductal carcinoma, the most common histologic type of carcinoma identified in men.

\section{CASE PRESENTATION}

A 66-year-old white man presented to his primary care physician complaining of a palpable mass in the left breast for 1 month. Routine digital mammographic images of both breasts (Figures 1 and 2) demonstrated a $2.4 \mathrm{~cm}$ mass within the subareolar left breast at the one o'clock position, which corresponded with the patient's palpable area of concern, with associated nipple inversion. The mass appeared spiculated and persisted on spot compression views. Sonographic evaluation of the left breast demonstrated a hypoechoic mass, measuring

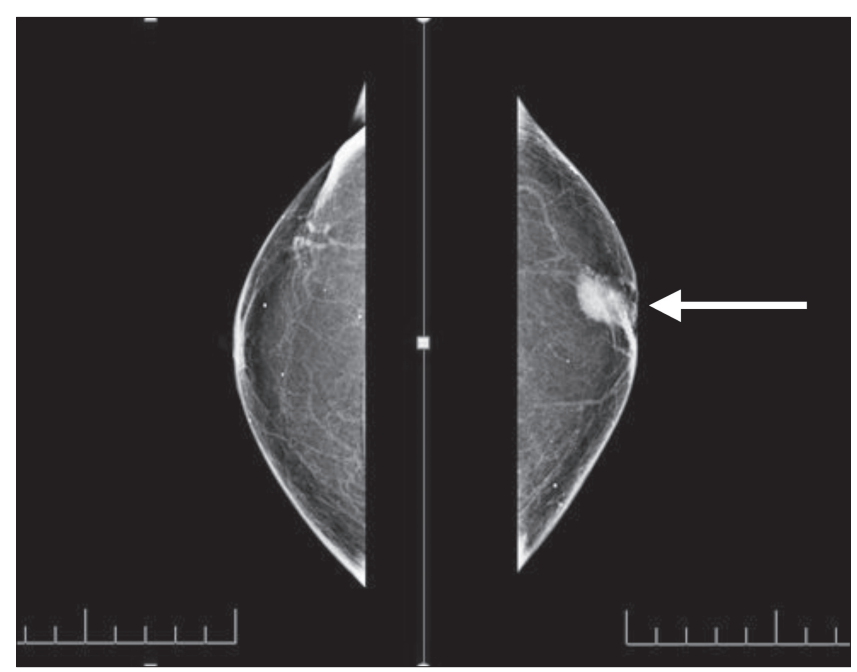

Figure 1. Routine craniocaudal views of both breasts demonstrate a $2.4 \mathrm{~cm}$ spiculated mass (arrow) within the subareolar left breast at the palpable area of concern. Associated nipple inversion was also identified.

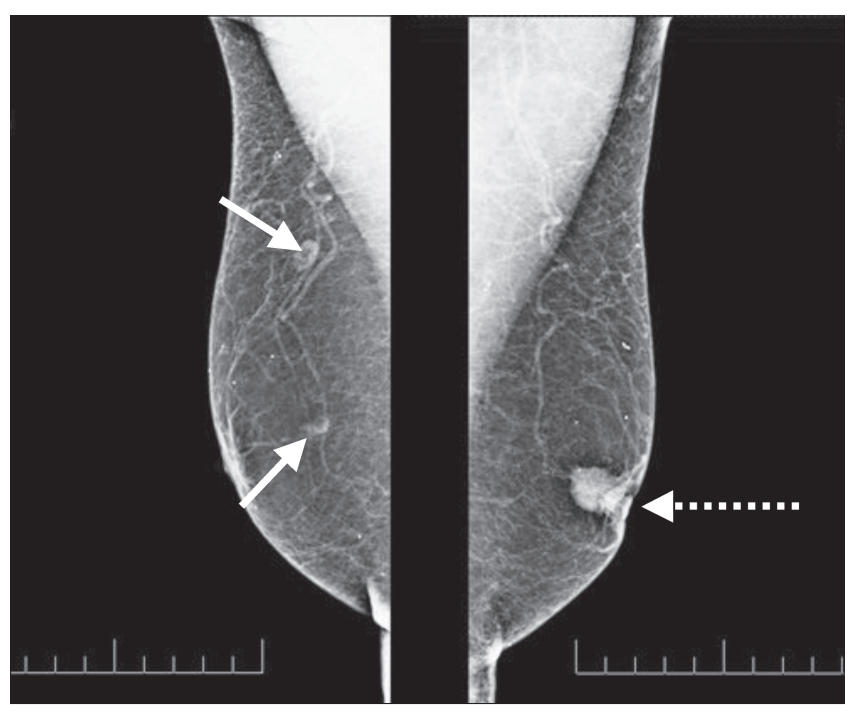

Figure 2. Mediolateral oblique views of both breasts demonstrate a spiculated $2.4 \mathrm{~cm}$ mass (dotted arrow) within the subareolar left breast at the palpable area of concern. Associated nipple inversion is also shown. Two subcentimeter lymph nodes are incidentally noted within the right breast (arrows).

$1.3 \times 1.3 \times 1.0 \mathrm{~cm}$, within the subareolar region (Figure 3). The patient underwent an excisional biopsy. The histologic diagnosis was high-grade invasive ductal carcinoma, and the patient was subsequently referred to an oncologist for further treatment.

\section{DISCUSSION}

Breast carcinoma in men represents approximately $1 \%$ of all breast cancers and $1 \%$ of all malignancies in men. The incidence of male breast cancer appears to be increasing (1). The risk increases with age, and the median age of diagnosis for men is later than that for women (2). There are several known risk factors for male breast carcinoma, including genetic predisposition, prior radiation exposure, alterations of the estrogen-testosterone ratio, and occupational hazards; our patient did not have any of these risk factors.

From the Department of Radiology, Baylor University Medical Center at Dallas. Corresponding author: Shannon Barry, MD, Department of Radiology, Baylor University Medical Center at Dallas, 3500 Gaston Avenue, Dallas, Texas 75246 (e-mail: shannon.barry4@gmail.com). 


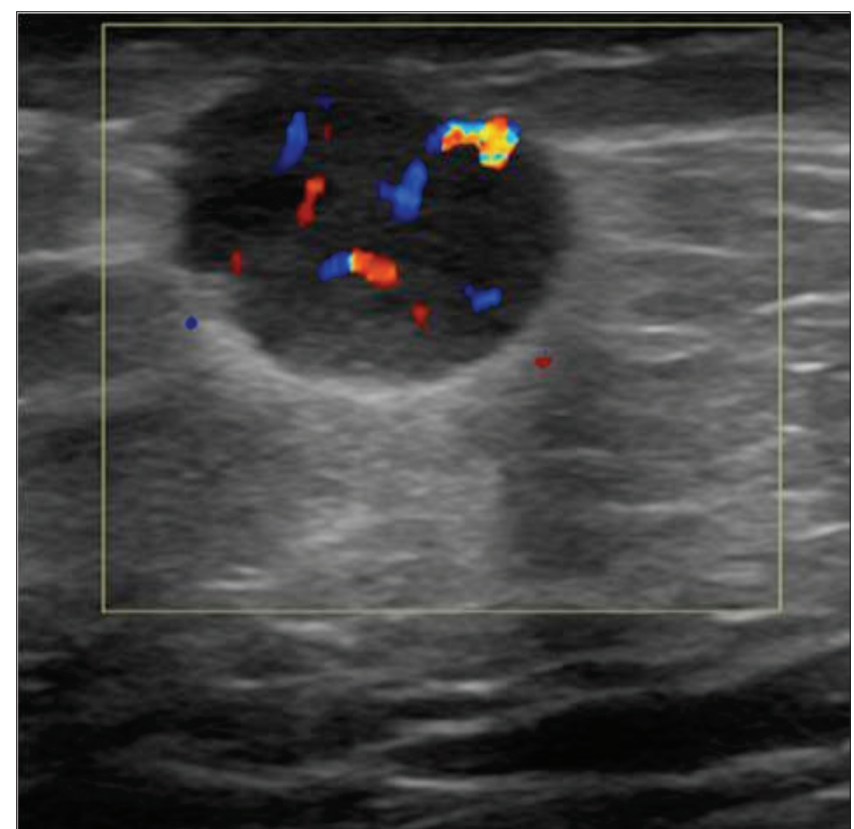

Figure 3. Sonographic image demonstrates a suspicious mass that measures $1.3 \times 1.3 \times 1.0 \mathrm{~cm}$ at the one $0^{\prime} \mathrm{clock}$ position near the nipple.

All of the histologic types of carcinoma identified in the female breast have been encountered in men, with invasive ductal carcinoma being the most common. Due to the absence of lobules in the normal male breast, invasive lobular carcinoma cases are seen infrequently. Bilateral breast cancer occurs in $<2 \%$ of male patients with breast cancer (3).

Over the past decade, there has been an increase in the number of imaging studies performed in men. These are largely performed in patients who present with complaints of a breast lump and/or breast pain. The combination of mammography with targeted breast ultrasound can be used to diagnose male breast carcinoma and has been reported to have a high sensitivity and specificity (4). There are limitations to these techniques, particularly in the male breast; for example, benign disease such as gynecomastia can mask underlying malignancy (5). Additionally, as male breast cancer typically occurs in an eccentric subareolar location, sonographic localization and evaluation of lesions can be complicated by the dense acoustic shadow of the nipple. Magnetic resonance imaging is being increasingly used in imaging the male breast and is an important diagnostic and problem-solving tool.

Currently, there are no guidelines regarding screening mammography in asymptomatic men at any age due to the rarity of male breast cancer. Current National Comprehensive Cancer Network guidelines for men with $B R C A$ mutations recommend consideration of baseline mammography followed by annual mammography in those men who are shown to have gynecomastia on the baseline study (6). Gynecomastia is an overdevelopment of the glandular tissue of the breast region in males. This is different from fat deposits in the breast, which are seen in obese men. Gynecomastia is seen in adolescent males during puberty and in older men. Coexistence of gynecomastia and ductal carcinoma, though rare, has been reported (7).

Treatment for male breast cancer depends on the histologic type of cancer as well as the degree of spread of disease at the time of diagnosis.

1. Kao L, Bulkin Y, Fineberg S, Montgomery L, Koenigsberg T. A case report: lobular carcinoma in situ in a male patient with subsequent invasive ductal carcinoma identified on screening breast MRI. J Cancer 2012;3:226230.

2. Saleh FM, Ansari NP. Invasive ductal carcinoma in a young male breast. Mymensingh Med J 2012;21(1):162-164.

3. Fidan E, Yildiz B, Ersoz S, Ozdemir F. Bilateral breast cancer: a male patient. Bratisl Lek Listy 2011;112(10):589-590.

4. Pant K, Dutta U. Understanding and management of male breast cancer: a critical review. Med Oncol 2008;25(3):294-298.

5. Westerland O, Shaw A, Howlett D. Re: Imaging male breast cancer. Clin Radiol 2012;67(5):512-513.

6. Korde LA, Zujewski JA, Kamin L, Giordano S, Domchek S, Anderson WF, Bartlett JM, Gelmon K, Nahleh Z, Bergh J, Cutuli B, Pruneri G, McCaskill-Stevens W, Gralow J, Hortobagyi G, Cardoso F. Multidisciplinary meeting on male breast cancer: summary and research recommendations. J Clin Oncol 2010;28(12):2114-2122.

7. Isley LM, Leddy RJ, Rumboldt T, Bernard JM. Asymptomatic incidental ductal carcinoma in situ in a male breast presenting with contralateral gynecomastia. J Clin Imaging Sci 2012;2:9. 